\title{
Selective induction of antibody effector functional responses using MF59-adjuvanted vaccination
}

\author{
Carolyn M. Boudreau, ${ }^{1,2}$ Wen-Han Yu, ${ }^{1}$ Todd J. Suscovich, ${ }^{1}$ H. Keipp Talbot,,${ }^{3,4}$ Kathryn M. Edwards, ${ }^{5}$ and Galit Alter ${ }^{1}$ \\ ${ }^{1}$ Ragon Institute of MGH, MIT and Harvard, Cambridge, Massachusetts, USA. ²PDD program in Virology, Division of Medical Sciences, Harvard University, Boston, Massachusetts, USA. ${ }^{3}$ Department of \\ Medicine, ${ }^{4}$ Department of Health Policy, and ${ }^{5}$ Department of Pediatrics, Vanderbilt University Medical Center, Nashville, Tennessee, USA.
}

\begin{abstract}
Seasonal and pandemic influenza infection remains a major public health concern worldwide. Driving robust humoral immunity has been a challenge given preexisting, often cross-reactive, immunity and in particular, poorly immunogenic avian antigens. To overcome immune barriers, the adjuvant MF59 has been used in seasonal influenza vaccines to increase antibody titers and improve neutralizing activity, translating to a moderate increase in protection in vulnerable populations. However, its effects on stimulating antibody effector functions, including NK cell activation, monocyte phagocytosis, and complement activity, all of which have been implicated in protection against influenza, have yet to be defined. Using systems serology, we assessed changes in antibody functional profiles in individuals who received H5N1 avian influenza vaccine administered with MF59, with alum, or delivered unadjuvanted. MF59 elicited antibody responses that stimulated robust neutrophil phagocytosis and complement activity. Conversely, vaccination with MF59 recruited NK cells poorly and drove moderate monocyte phagocytic activity, both likely compromised because of the induction of antibodies that did not bind FCCR3A. Collectively, defining the humoral antibody functions induced by distinct adjuvants may provide a path to designing next-generation vaccines that can selectively leverage the humoral immune functions, beyond binding and neutralization, resulting in better protection from infection.
\end{abstract}

\section{Introduction}

Seasonal influenza infects $10 \%-20 \%$ of the worldwide population each year, with a mortality rate of $0.1 \%-0.2 \%$, and increased mortality in the elderly $(1,2)$. By contrast, avian influenza viruses that are endemic to global fowl populations, such as H5N1, infect significantly fewer humans per year, but have a much higher mortality rate (approximately $60 \%$ for H5N1) (3). Although these avian viruses cannot effectively spread from human to human, it has been suggested that we are on the precipice of highly lethal avian influenza viruses evolving to allow human-to-human transmission (4). This could result in the rapid global spread of the virus, resulting in the next influenza pandemic $(3,5)$. To mitigate this concern, pandemic influenza vaccines are urgently needed. These vaccines will need to provide rapid and robust protection against the potential threat of an avian influenza pandemic. Current influenza vaccine design strategies, in the best-case scenario, require 5-6 months from determination of the viral sequence to full-scale vaccine production, time that is not available during a pandemic. In addition, vaccine strategies that induce equivalent immunity using less antigen, termed antigen sparing, are needed to expand the quantity of vaccine available for the global population.

Adjuvants have the potential to decrease antigen quantity requirements and to improve vaccine effectiveness by enhancing antigen delivery to antigen-presenting cells and by shaping the functional potency of the vaccine-induced immune response

Conflict of interest: The authors have declared that no conflict of interest exists. Copyright: () 2020, American Society for Clinical Investigation.

Submitted: April 12, 2019; Accepted: October 15, 2019; Published: December 17, 2019. Reference information: / Clin Invest. 2020;130(2):662-672.

https://doi.org/10.1172/JCI129520.
(6). Currently, 6 adjuvants have been approved for use in licensed human vaccines, of which 4 have been assessed for use in influenza vaccines (7). Among these adjuvants, MF59 is an oil-in-water emulsion licensed for use in seasonal influenza vaccines (8). In seasonal influenza vaccination, MF59 is known to induce monocyte and granulocyte chemoattractants (9); to alter the antibody-mediated immune response by inducing heterotypic antibody responses, increasing the breadth and affinity of the vaccine-induced antibodies; and to increase neutralizing antibody responses $(5,10$, 11). Furthermore, MF59 exhibits antigen-sparing activity, driving robust neutralizing antibody responses at 3-fold lower doses of $\mathrm{H} 5$ antigen (11). However, despite its potent immunological effects, the addition of MF59 does not consistently afford equally potent enhanced protection from seasonal influenza infection to the level that would be predicted by the increased antibody responses (12, 13). These data raise the possibility that MF59 is able to drive enhanced vaccine-induced immunity, but it may fail to raise a key humoral immune function required to achieve protection.

Although the development of broadly neutralizing antibody responses against influenza remains a critical goal, emerging evidence suggests that extra-neutralizing antibody functions also play an important role in protection from infection (14-23). Studies with passive transfer of neutralizing monoclonal antibodies in mice have shown that $\mathrm{Fc} \gamma$ receptors are required for protection from lethal infection, via mechanisms that do not block viral entry (18). In addition to binding pathogens and preventing infection of cells, antibodies mediate an array of functions, including complement activation and lysis; phagocytosis by granulocytes, monocytes, and dendritic cells; NK cell activation; and dendritic cell maturation and antigen presentation (24-26). Influenza-specific antibodies have been shown to mediate macrophage phagocy- 
A

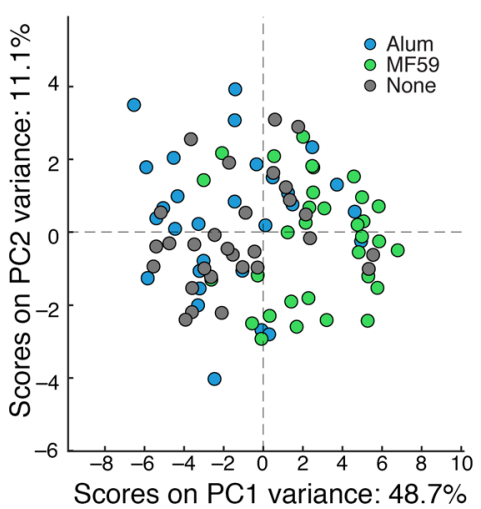

B

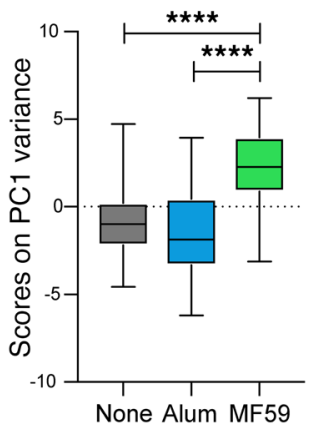

C

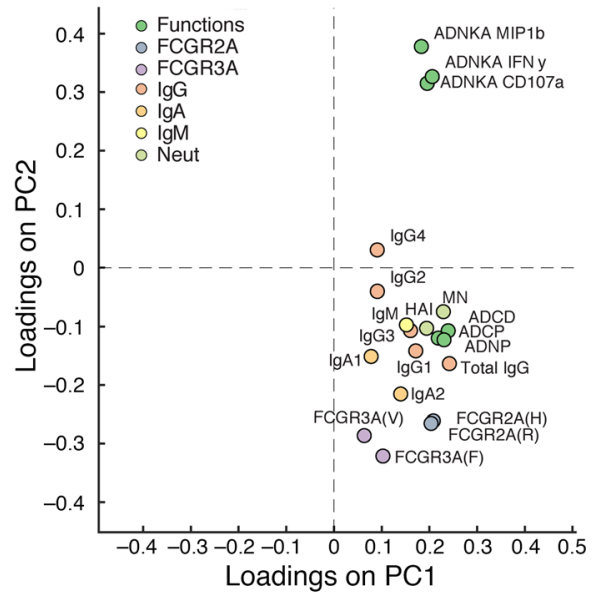

Figure 1. MF59 significantly alters the functional humoral profile after H5-vaccination. Antibodies against $\mathrm{H} 5$ were compared for 90 vaccinees who received $15 \mu$ recombinant $\mathrm{H} 5$ unadjuvanted (none), with alum, or with MF59. A principal components analysis (PCA) was used to identify differences in the functional responses of vaccinees by adjuvant. (A) All H5-specific systems serology measurements (antibody isotypes, FCGR binding, and antibodydependent functions) from post-vaccine day 56 samples were included in the PCA biplot, where dots represent individual samples (blue, alum; green, MF59; gray, none). (B) Scores along the first principal component (PC1), which captures the greatest separation between samples, were plotted. Error bars show minimum to maximum. Differences were assessed using a Mann-Whitney $U$ test. ${ }^{* * *} P<0.0001$. (C) Loadings in the PCA biplot are shown. Features are located in the loadings plot where they are enriched in samples in $\mathbf{A}$.

tosis $(20,27)$, neutrophil production of reactive oxygen species (20), cellular cytotoxicity (21), and complement deposition (16, 17, 27, 28). However, data using broadly neutralizing monoclonal antibodies suggest that NK cell-driven antibody-dependent cellular cytotoxicity (ADCC) may represent a key protective function against influenza $(18,19,29)$.

Previous studies have demonstrated that MF59 can increase the breadth of the heterosubtypic immune responses and neutralizing titers; the goal of this study was to systematically probe the functional effect of MF59-induced immunity when administered in a potential pandemic avian influenza vaccine. Using an unbiased, high-throughput systems serology (30) platform, we observed increased antigen-specific antibody functionality in serum from individuals who received MF59-adjuvanted vaccinations, with the notable exception of titer-dependent increases in NK cell activation or monocyte phagocytic activity, highlighting the robust induction of humoral immune responses programmed to induce selective antibody effector functions.

\section{Results}

MF59 induces a unique functional humoral response. Emerging data suggest that adjuvants may induce higher antibody titers and enhanced affinity maturation and may shape the Fc-profile of antibodies (31). To initially define whether adjuvants also shape influenza-specific humoral immune responses, we applied a systems serology (30) approach to broadly and comprehensively profile the functional and Fc-biophysical profiles of antibodies generated by vaccinees receiving pandemic influenza $\mathrm{H} 5$ immunization alone or adjuvanted with MF59 or alum. Using this suite of functional and biophysical assays, over 50 data points were collected per subject, encompassing the ability of antibodies to recruit NK cells, monocytes, neutrophils, and complement. In addition, differences in the subclass and isotype selection and Fc $\gamma$-receptor binding were analyzed across H5-specific immune responses. To determine whether induced profiles were any different at the peak response, 56 days after initial vaccination/28 days after the second dose, a principal components analysis (PCA) was used to assess adjuvant effects (Figure 1A). Although the alum-adjuvanted and unadjuvanted vaccine groups overlapped and were nearly indistinguishable, the majority of the MF59 group clearly separated, exhibiting a unique antibody Fc-profile. To further quantify the significance of the difference between MF59 and alum or unadjuvanted vaccines, scores along the first principal component (PC1) were analyzed (Figure 1B). As demonstrated in the PCA plot (Figure 1A), serum samples from individuals receiving MF59 exhibited a unique and separate response when compared with either the alum-adjuvanted or unadjuvanted groups. At baseline (day 0), the responses to $\mathrm{H} 5$ were indistinguishable from the other groups (Supplemental Figure 1; supplemental material available online with this article; https://doi.org/10.1172/JCI129520DS1). These data suggest that, unlike alum, MF59 induces a significantly different functional humoral immune response compared with unadjuvanted vaccination (Figure 1A).

To gain a deeper sense of the differences in the MF59induced multivariate profile, a loadings plot was generated showing the specific features that were differentially enriched within each vaccine group profile (Figure 1C). Features cluster on the loadings plot in the same region as the samples in which they are enriched. Along these lines, nearly all features were enriched in the MF59-adjuvanted vaccine recipient profiles, except for the antibody-dependent NK cell activation (ADNKA) features, which clustered in a separate region of the graph. Consistent with previous studies (11, 32), H5-specific IgG titers were enriched in the MF59 group. Antibody complement activity, the ability to drive monocyte phagocytosis, and neutrophil phagocytosis were also enriched in MF59 profiles when compared with the 2 other groups 
A

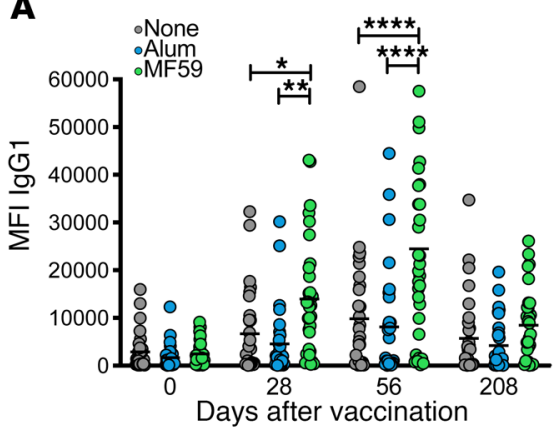

B

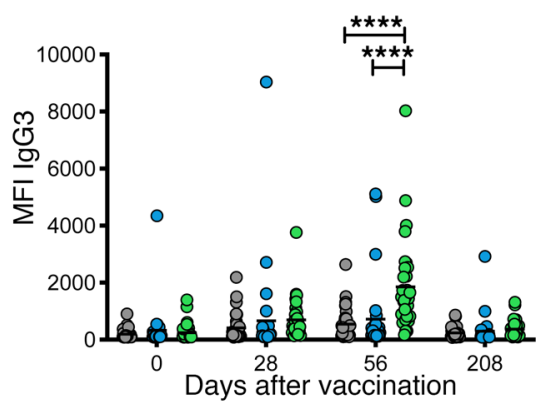

C

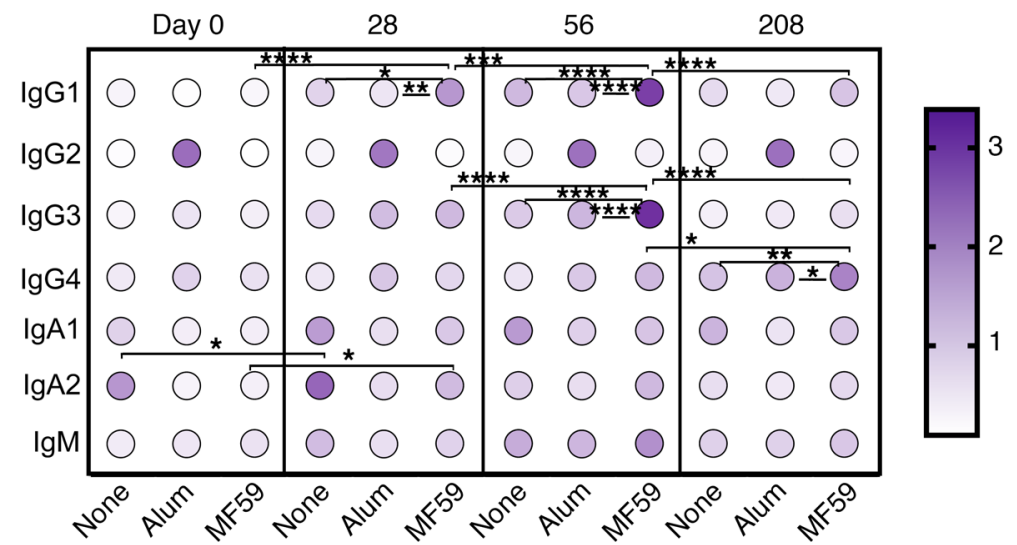

Figure 2. MF59 selectively enhanced functional antibody subclass levels after $\mathrm{H} 5$ immunization. (A) The dot plot shows H5-specific IgG1 titers as measured by Luminex in all 3 vaccine groups over 4 time points. Each dot represents the average of 2 replicates for 1 serum sample. Bar represents group mean. (B) The dot plot shows H5-specific IgG3 titers as measured by Luminex in all 3 vaccine groups over 4 time points. Each dot represents the average of 2 replicates for one serum sample. Bar represents group mean. (A and B) Significance was determined by 2-way ANOVA followed by Tukey's multiple comparisons test. Significance is noted only within time points. ${ }^{*} P<0.05,{ }^{* *} P<0.01,{ }^{* *^{* *}} P$ $<0.0001$. (C) Titers for other antibody isotypes and subclasses across all 3 vaccine groups are shown in the heatmap over 4 time-points, depicted as values normalized by dividing by row mean. Significance was determined on raw values by 2 -way ANOVA followed by Tukey's multiple comparisons test. ${ }^{*} P<$ $0.05,{ }^{* *} P<0.01,{ }^{* * *} P<0.001,{ }^{* * *} P<0.0001$. and contributed to the separation across the vaccine samples. Moreover, limited shifts were observed with IgG4, IgG2, and IgM responses that remained in the middle of the loadings plot (Figure $1 C)$, whereas more pronounced shifts were observed in total $\operatorname{IgG}$, surpassed by Fc-receptor binding antibodies, pointing to a combination of qualitative and quantitative, rather than strictly titerbased, changes in H5-specific immunity. Thus, beyond strictly quantitative differences, MF59 induced a qualitatively different antibody profile compared with the vaccine administered with alum or no adjuvant.

MF59 enhances highly functional antibody subclass levels. Previous studies have demonstrated that MF59 can increase antibody titers, but it is not clear whether alum and MF59 also drive differential isotype and subclass profiles. Although alum induced $\operatorname{IgG1}$ (Figure 2A) and IgG3 antibodies (Figure 2B), the levels were not different from those induced using the unadjuvanted vaccine. Conversely, MF59 drove significantly higher levels of total antigenspecific IgG1 at days 28 and 56 (Figure 2A), and higher IgG3 responses at day 56 (Figure 2B), consistent with previously reported data $(11,32)$. Despite this significant increase in IgG1 and IgG3 antibodies, these responses were largely lost by day 208 , when all the titers returned to near-baseline levels irrespective of vaccine group.

Beyond IgG subclass level differences, all vaccine strategies slightly, but not significantly, raised IgM levels by day 56 (Figure $2 \mathrm{C}$ and Supplemental Figure 2D). The unadjuvanted vaccine also tended to drive elevated levels of IgA1 and IgA2 H5-specific antibodies, although the difference was not always significant. Alum did not differ globally from the unadjuvanted vaccine profile: IgA1 was induced in the nonadjuvanted group at days 28 and 56 but not in the alum-adjuvanted group (Figure $2 \mathrm{C}$ and Supplemental
Figure 2E). In contrast, MF59 drove significantly higher levels of functionally potent $\operatorname{IgG} 1$ and $\operatorname{IgG} 3$ responses (Figure 2, A and B). MF59 did not drive significantly increased IgA in the serum when compared with unadjuvanted or alum-adjuvanted vaccination. These data suggest that all vaccine groups recruited IgM responses; however, adjuvants shifted the unadjuvanted vaccine profile away from IgA-dominated responses. Yet, unlike alum, MF59 drove an enhanced functional IgG1- and IgG3-dominated response, further suggesting that, in addition to quantitative changes, MF59 also induces a qualitative change in vaccine-induced immunity toward more functional IgG subclasses. Despite the more potent functional subclass induction, MF59 did not show evidence of enhanced durability of the vaccine response.

MF59 selectively induces antibody-dependent innate immune functions. Given the emerging appreciation for a critical role for antibody effector function in protection against influenza $(15,19)$, we next probed whether the observed changes in antibody titer and isotype/subclass selection differences also tracked with changes in antibody effector functions. Previous studies demonstrated that the rapid increase in MF59-induced titers by day 28 was associated with a delayed increase in hemagglutination inhibition (HAI) and microneutralization at day 56 that declined to baseline levels by day 208 (Figure 3, A and C). To gain a deeper appreciation for the relationship between HAI and vaccine-induced titers, HAI was normalized to titers at each time point (Figure 3B). Interestingly, $\mathrm{HAI} /$ titer ratios were consistent across all vaccine arms, with the exception of day 208, when HAI activity was disproportionately lower than H5-titers in the MF59 group. This selective reduction in the HAI/titer ratio points to a selective loss of HAI-mediating antibodies among MF59 vaccinees at this late time point (Figure 3B). In 

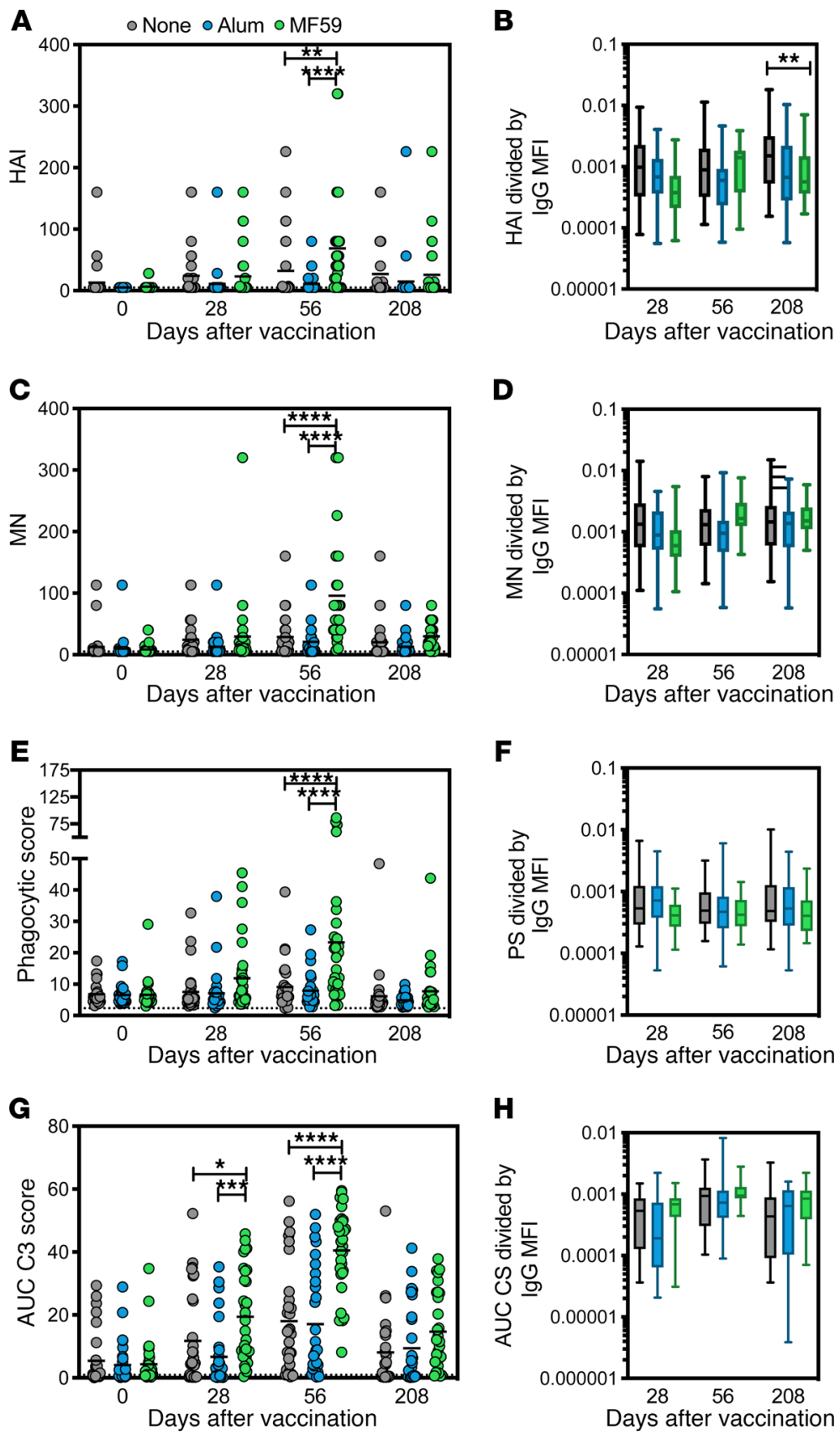

Figure 3. MF59 induces neutralization and specific antibody-dependent innate immune functions along with IgC1 titers. (A) HAl activity for serum samples in all 3 vaccine groups over 4 time points (11). Each dot represents the average of 2 replicates for 1 serum sample. For all dot plots, bar shows group mean. The dotted line indicates the limit of detection. (B) HAl activity divided by total IgG Luminex MFI as reported in Figure 2 for all 3 vaccine groups during the 3 post-vaccine time points. For all box plots, error bars show minimum to maximum. (C) Microneutralization (MN) activity for serum samples (11). Each dot represents the average of 2 replicates for 1 serum sample. The dotted line indicates the limit of detection. (D) MN activity divided by total IgG Luminex MFI for all 3 vaccine groups. (E) Average ADNP activity for serum samples across 2 healthy WBC donors. Each dot represents the average of 2 replicates for 1 serum sample. The dotted line indicates flu-negative serum background. (F) ADNP activity divided by total IgC Luminex MFI for all 3 vaccine groups. (C) ADCD by serum samples. Each dot represents the average of 2 replicates for 1 serum sample. The dotted line indicates the flu-negative serum background. Values are indicated as the average area under the curve of 3 serum dilutions run in 2 independent replicates. (H) ADCD activity divided by total IgG Luminex MFI. Significance was tested by 2-way ANOVA followed by Tukey's multiple comparisons test. Significance was only noted within time point. ${ }^{*} P<0.05$, ${ }^{* *} P<0.01,{ }^{* *} P<0.001$, ${ }^{* * *} P<0.0001$. contrast, no differences between groups were observed in the microneutralization/titer ratios at all time points, highlighting the direct relationship between neutralization and titers across all vaccine arms (Figure 3D). These data point to a titer-based increase in HAI and microneutralization across all vaccine arms, but a sharper decline in HAI, unlinked from titer, in the MF59-immunized group at day 208 .

To probe whether the same kinetics and titer-based relationships emerged for $\mathrm{Fc}$-effector functions, we assessed the ability of vaccine-induced antibodies to drive antibody-dependent neutrophil phagocytosis (ADNP), antibody-dependent complement deposition (ADCD), antibody-dependent cellular phagocytosis (ADCP), and antibody-dependent NK activation (ADNKA). A robust ADNP-inducing antibody response was observed after MF59-adjuvanted immunization (Figure 3E), following similar kinetics to those observed for both HAI and microneutralization in the MF59 arm (Figure 3, A and C). Conversely, these antibodies were significantly lower in the alum and unadjuvanted arms (Figure 3E). ADNP peaked at week 56 in MF59 vaccinees and declined to baseline levels by day 208, similar to changes observed in antibody titers. Moreover, normalization of ADNP by titer demonstrated equivalent levels of ADNP-per-titer activity across all vaccine arms, highlighting that ADNP activity increased in an equally titer-dependent manner over time irrespective of adjuvant (Figure 3F).

Similar to ADNP, MF59-adjuvanted vaccinees exhibited a significant increase in ADCD responses when compared with unadjuvanted and alum-adjuvanted vaccinees. This increase occurred earlier than peak titers, with a significant increase in complement-fixing activity by day 28 , but a more significant increase of complement-fixing antibodies by day 56 after vaccination (Figure 3G). ADCD-inducing antibodies declined by day 208 but remained slightly higher in the MF59-vaccinated group (Figure 3G). Normalization of ADCD to titer demonstrated the strong and equal association between titer and function across all vaccine groups, wherein the ADCD/ titer ratio did not change between groups during the vaccine study, suggesting that ADCD simply increased in a titer-dependent manner across all vaccine strategies (Figure 3H), similar to HAI and microneutralization (Figure $3, \mathrm{~B}$ and D).

ADCP increased in a similar manner to ADCD but remained significantly elevated in the MF59 group at day 208 (Figure 4A), despite the return to equal titers between vaccine arms (Figure 2A). However, normalization of ADCP to titer highlighted a disproportionate increase in titer compared with ADCP activity in the MF59 group, in such a way that lower levels of ADCP were induced per titer level in the MF59 group compared with the unadjuvanted or alum-adjuvanted groups at days 28 and 56 (Figure 4B). These data suggest that HAI, microneutralization, ADNP, and ADCD were induced 
A

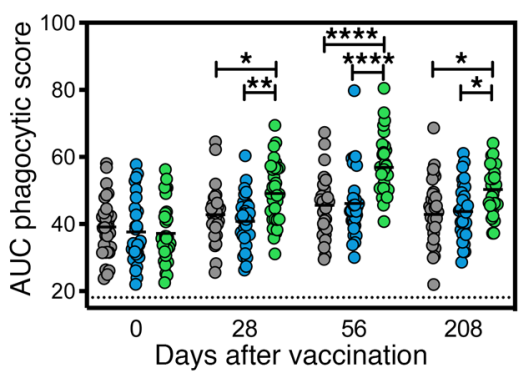

C

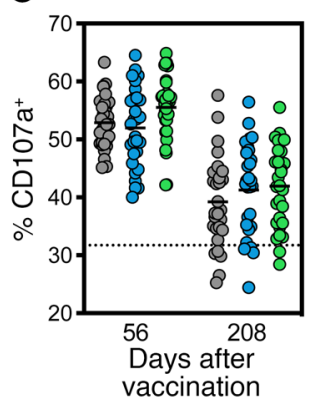

D

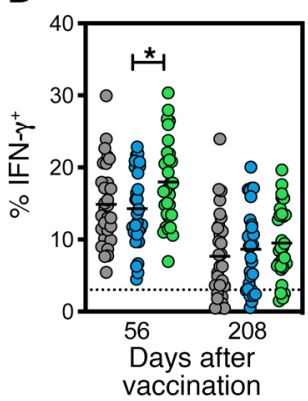

G

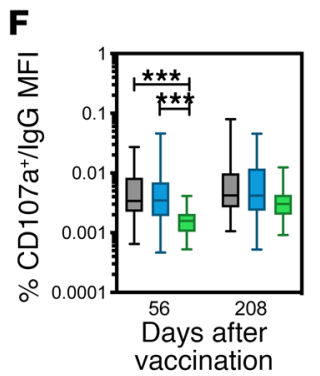

B

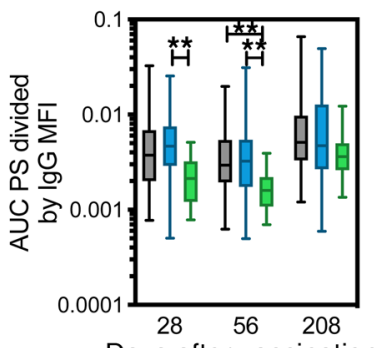

Days after vaccination

E

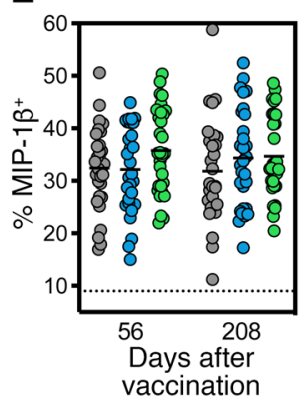

H

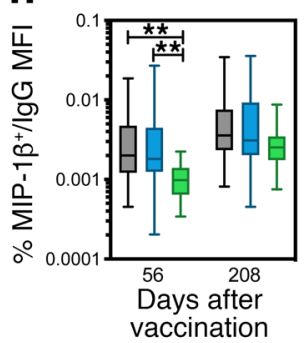

Figure 4. MF59 induces higher titers, but not antibody-dependent monocyte or NK cell functions. (A) ADCP activity for serum samples in all 3 vaccine groups over 4 time points (11). Each dot represents the average of 2 replicates for 1 serum sample. For all dot plots, bar shows group mean. The dotted line indicates the flu-negative serum background. Values are indicated as the area under the curve of 3 serum dilutions run in 2 independent replicates. (B) ADCP activity divided by total IgC Luminex MFI. (C-E) ADNKA activity by average percentage of NK cells expressing each of 3 markers, CD107a (C), IFN- $\gamma$ (D), and MIP-1 $\beta$ (E), for 2 healthy NK cell donors. The dotted line indicates flu-negative serum background. (F-H) ADNKA activity for 3 markers divided by total IgG Luminex MFI for all 3 vaccine groups. Significance was tested by 2-way ANOVA followed by Tukey's multiple comparisons test. Significance was only noted within time point. ${ }^{*} P<0.05$, ${ }^{*} P<0.01$, ${ }^{* * *} P<0.001,{ }^{* * *} P<0.0001$.

in a titer-dependent manner, whereas MF59-induced antibodies possessed less ADCP per antibody generated when compared with responses in other vaccine arms, pointing to a functional divergence between the overall quantity and quality of adjuvanted vaccineinduced antibodies.

Given the emerging appreciation for the importance of ADCC activity in protection against influenza (33), we profiled differences in the NK cell-activating ability of H5-specific antibodies after unadjuvanted, alum-adjuvanted, and MF59-adjuvanted vaccination at days 56 and 208 (Figure 4, C-E). H5N1-vaccine-induced antibodies were able to drive NK cell degranulation (upregulation of CD107a, a marker of NK cell cytotoxicity; ref. 34) and NK cell chemokine secretion (macrophage inflammatory protein $1 \beta$ [MIP1 $\beta]$ ), but these responses were not different across the 3 vac- cine groups (Figure 4, C and E), despite observed titer differences (Figure 2A). MF59 induced H5-specific antibodies able to stimulate more NK cell IFN- $\gamma$ secretion (Figure 4D). However, normalization of NK cellactivating antibody levels to titer revealed disproportionately lower NK/titer ratios among MF59 vaccinees, across all NK cell functions (Figure 4, F-H). These results suggest that despite the robust induction of antibody titers, HAI, microneutralization, ADCD, and ADNP, MF59-induced antibodies possessed lower NK cell-activating activity on a per-antibody level compared with those induced by the vaccine alone or delivered with alum. Thus, MF59-adjuvanted vaccination may result in weaker induction of NK cell-activating humoral immune responses on a per-antibody level.

Taken together, these data point to a potent effect of MF59 in driving H5-specific functional antibodies, but with a reduced capacity to fully harness NK cell and monocyte functional responses.

MF59 induces antibodies with differential lowaffinity Fcy-receptor binding profiles. To gain further insights into the specific mechanics that could contribute to differential functional responses, we next aimed to explore changes in vaccine-induced antibody interactions with Fc-receptors involved in directing innate immune effector function. Specifically, beyond changes in vaccine-induced titers, we examined the ability of $\mathrm{H} 5$-specific antibodies to interact with the 2 low-affinity Fc $\gamma$-receptors involved in inducing phagocytosis (FCGR2A) and NK cell cytotoxicity (FCGR3A). In agreement with the functional data, all vaccine groups experienced an increase in FCGR2A binding (Figure 5A) at day 28 and day 56; however, subjects receiving MF59 experienced a significantly higher increase in FCGR2A binding. Moreover, all FCGR2A binding antibodies had declined at day 208, but the levels in MF59 vaccinees remained elevated, consistent with enhanced ADCP activity (Figure 3E) over time and unlinked from changes in overall H5-specific antibody titers (Figure 2A). Conversely, despite the increase in IgG1 and IgG3 titers, only a slightly insignificant increase in FCGR3A binding antibodies at day 28 was observed in MF59adjuvanted vaccine recipients compared with other groups. However, FCGR3A-binding antibody levels declined rapidly across all groups over time (Figure 5B). Moreover, analysis of vaccine-induced binding profiles across high- and low-affinity variants of FCGR2A and FCGR3A showed consistent profiles: FCGR2A binding was clearly augmented across both allotypes, with a preferential significant increase among MF59 vaccinees (Figure 5C and Supplemental Figure 4). Similarly, no evidence of an increase in FCGR3A binding was observed across both FCGR3A variants $(35,36)$, highlighting the consistent lack of FCGR3A binding in all H5N1 vaccinees (Figure 5C and Supplemental Figure 4). Given that NK cells and monocytes express FCGR3A (35), unlike neutrophils, these data are consistent with a lack of a NK cell-activating and monocyte phagocytic activity. 
A

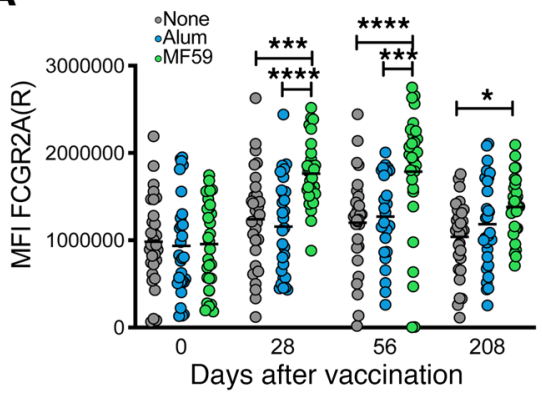

B

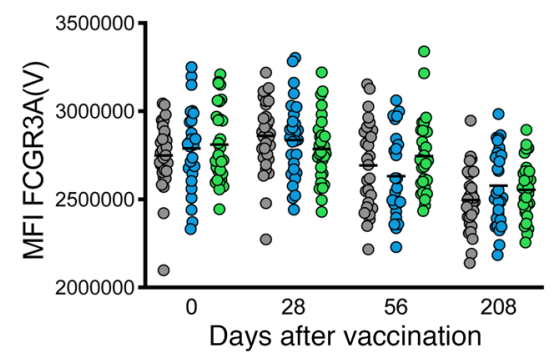

Figure 5. MF59 increases enhanced Fc $\gamma$ RIla, but not FcyRIIIa, H5-specific binding antibodies.

(A) The dot plot shows $\mathrm{H} 5$-specific antibody binding to FCCR2A(R) as measured by Luminex in all 3 vaccine groups over 4 time points. Each dot represents the average of 2 replicates for 1 serum sample. Bar shows group mean. (B) The dot plot shows $\mathrm{H} 5$-specific antibody binding to FCGR3A(V) as measured by Luminex bead array in all 3 vaccine groups over 4 time points. Each dot represents the average of 2 replicates for 1 serum sample. Bar shows group mean. (A and $\mathbf{B}$ ) Significance was determined by 2-way ANOVA followed by Tukey's multiple comparisons test. Significance is noted only within time points. ${ }^{*} P<0.05$, ${ }^{* * *} P<0.001$, ${ }^{* * *} P<0.0001$. (C) FCR binding for both allotypes of FCGR2A and FCGR3A for other antibody isotypes in all 3 vaccine groups over 4 time points are shown in a heatmap as values normalized by dividing by the row mean. Significance was determined on raw values by 2-way ANOVA followed by Tukey's multiple comparisons test. ${ }^{*} P<0.05,{ }^{* *} P<0.01$ ${ }^{* * *} P<0.001,{ }^{* * *} P<0.0001$.

MF59 overcomes natural preexisting HA-specific antibodies. Previous data suggest that preexisting influenza immunity shapes the induction of the humoral immune response, even to distant HAs (37), so we sought to determine whether the functional profiles of vaccine-induced antibodies were differentially influenced by natural preexisting humoral immune responses. We hypothesized that individuals with generally higher levels of phagocytic antibodies in response to seasonal influenza may leverage these responses more effectively after $\mathrm{H} 5 \mathrm{~N} 1$ avian influenza vaccination. H1 responses were used as a proxy for seasonal influenza responses. Vaccinees within each group were thus split into tertiles based on their preexisting $\mathrm{H} 1$-specific functional responses at baseline (Figure 6, A, D, and G). H5-specific responses were then compared across each group at peak immunogenicity (day 56). MF59 increased the $\mathrm{H} 5$-specific ADCP response at the peak time point in all 3 groups, suggesting that preexisting H1-specific ADCP function did not influence the induction of H5-specific ADCP (Figure 6B). No significant differences were observed in $\mathrm{H} 5$-specific function among low, medium, and high baseline responders within the 3 vaccine groups (Figure 6B). This points to a limited effect of previous influenza exposure in influencing antibody effector function to a novel influenza hemagglutinin. Importantly, this upregulation of phagocytic function, specifically in the MF59 group, was not associated with a concomitant increase in H1-specific functional activity (Figure 6C), further demonstrating the specific recruitment of $\mathrm{H} 5$-specific immune responses, rather than the corecruitment of preexisting H1-specific immune responses.

Using a similar grouping strategy, no difference was observed in H5-specific neutrophil phagocytic activity (Figure 6E) or complement activity (Figure 6H) across the $\mathrm{H} 1$ tertiles among the 3 vaccine groups, highlighting the lack of an impact of preexisting potentially cross-reactive H1-specific immunity. In contrast, a trend toward higher ADNP- and ADCD-recruiting cross-reactive H1-specific responses was detected in subjects with the highest levels of preexisting H1-specific immunity (Figure 6, F and I). Taken together, these data suggest that preexisting cross-reactive immunity does not influence the induction of functional antibodies to diverging HAs (H5N1) induced by MF59.

\section{Discussion}

Over 100 years since the Spanish flu pandemic of 1918, strategies to prepare for an emerging pandemic influenza strain, such as H5N1 avian influenza, are still inadequate. Several strategies have been proposed, including the following: (a) the acceleration of vaccine production pipelines (38), (b) the development of novel antigens able to drive immunity to sites of vulnerability like the HA stem (39), (c) the identification of immune correlates to induce targeted vaccine immune functions (40), (d) the generation of antigen-sparing vaccine approaches able to ensure sufficient material to cover the global population (38), and (e) the development of strategies to enhance the protective activity of vaccine-induced humoral immunity. Specifically, adjuvant technologies have been proposed to address the latter 3 efforts. Alum is the most widely used adjuvant and the standard comparator for novel adjuvant formulations, but did not satisfy these goals for avian influenza vaccination, consistent with previous studies (41, 42). MF59, in contrast, exhibits antigen-sparing and immune-boosting functions and has shown promise in seasonal and avian influenza vaccines by significantly increasing antibody response titers after vaccination $(5,10,32,43)$. Despite these increases in titer, MF59-adjuvanted vaccination still provided only partial protection from seasonal infection in large studies $(12,13)$. Specifically, although MF59 conferred much higher protection among the youngest age group (6-23 months) compared 
A

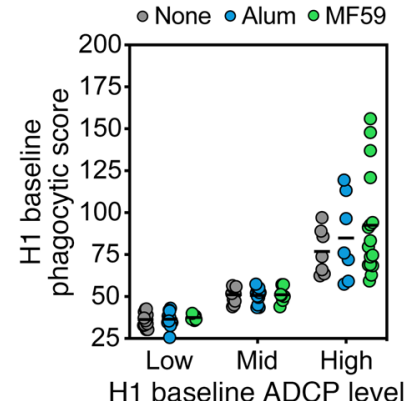

D

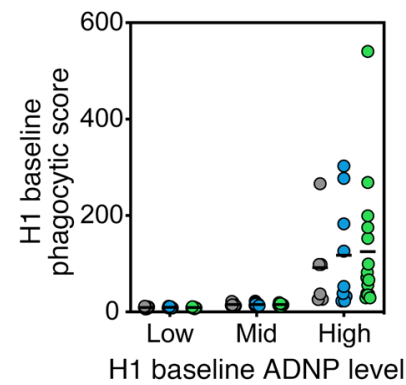

G

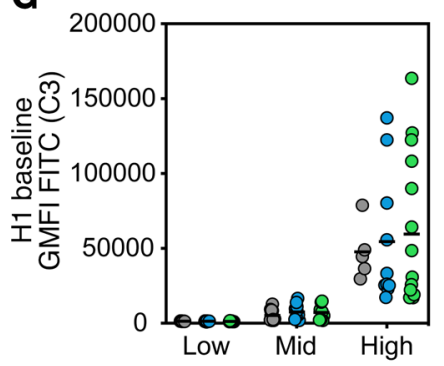

H1 baseline ADCD level

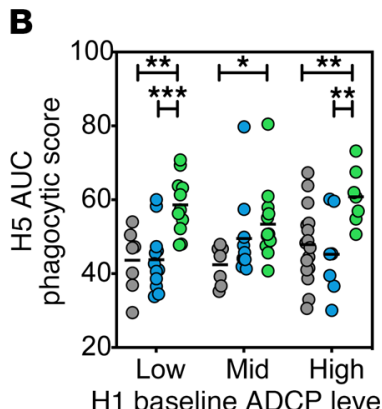

C

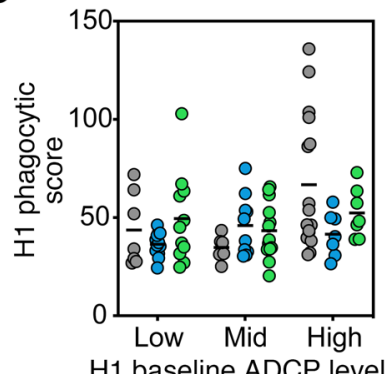

E

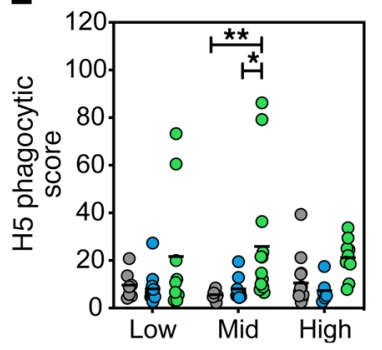

$\mathrm{H} 1$ baseline ADNP level

$\mathbf{F}$

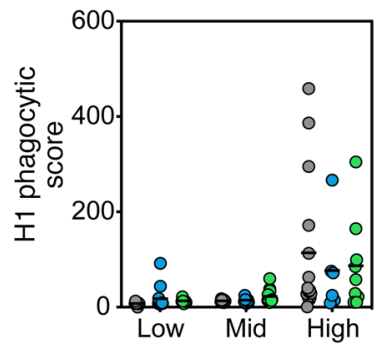

$\mathrm{H} 1$ baseline ADNP level

I

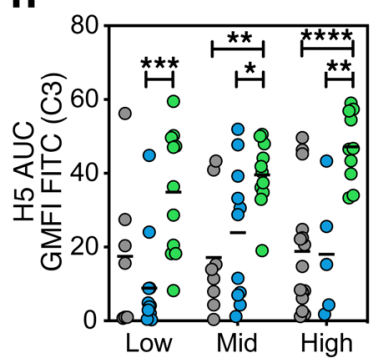

$\mathrm{H} 1$ baseline $A D C D$ level

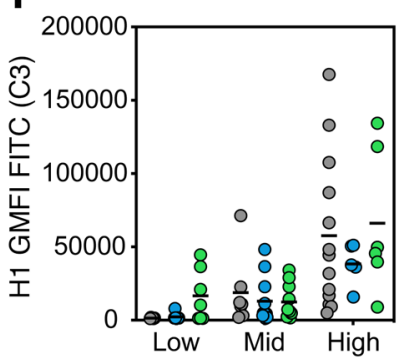

$\mathrm{H} 1$ baseline ADCD level

Figure 6. MF59-induced antibody functionality is not influenced by prevaccination H1-specific immunity. (A) Samples were grouped into low, mid, or high based on their baseline (day 0) H1-specific ADCP. The dot plot indicates grouping strategy of baseline H1-specific ADCP among each vaccine group. (B) H5-specific ADCP at peak immunogenicity (day 56) for H1 baseline ADCP groups. (C) H1-specific ADCP at peak immunogenicity for each baseline reactivity group by vaccine adjuvant. (D) Samples were grouped into low, mid, or high based on their baseline (day 0) H1-specific ADNP. The dot plot indicates the grouping strategy of baseline H1-specific ADNP among each vaccine group. (E) H5-specific ADNP at peak immunogenicity (day 56) for H1 baseline ADNP groups. (F) H1-specific ADNP at peak immunogenicity for each baseline reactivity group by vaccine adjuvant. (C) Samples were grouped into low, mid, or high based on their baseline (day 0) H1-specific ADCD. Dot plot indicates grouping strategy of baseline H1-specific ADCD among each vaccine group. (H) H5-specific ADCD at peak immunogenicity (day 56) for H1 baseline ADCD groups. (I) H1-specific ADCD at peak immunogenicity for each baseline reactivity group by vaccine adjuvant. For all dot plots, each dot represents the average of 2 replicates for 1 serum sample, and bar shows group mean. Significance was tested by 2-way ANOVA followed by Tukey's multiple comparisons test and is noted only within time point. ${ }^{*} P<0.05,{ }^{* *} P<$ $0.01,{ }^{* *} P<0.001,{ }^{* * *} P<0.0001$.

with unadjuvanted vaccination, this level of protection was not observed across the whole study population (12). Thus, the level of protection did not reach the level expected to be conferred by enhanced antibody titers, pointing to a critical disconnect between the quality and quantity of influenza-specific humoral immunity. This is reflected in the data presented here, wherein MF59 significantly augmented the H5-specific antibody titer but did not equally enrich for all antibody-dependent innate immune cell functions in a titer-dependent manner; notable gaps were observed in monocyte phagocytic and NK cell functions.

Given our emerging appreciation for the importance of adjuvants in shaping antibody function (44), here we aimed to deeply profile and compare the functional quality of the antibody response driven by distinct adjuvants. We found that alum slightly enhanced the Th1 character of the humoral immune response, marginally moving the isotype profile away from IgA1 and IgA2 (Figure 2C and Supplemental Figure 2, E and F), and MF59 drove significant IgG1 and IgG3 responses able to drive highly neutrophil phagocytic- and complement-inducing responses. IgG and IgA antibodies are critical for the control of influenza infection, with mucosal and serum IgA complementing IgG in the serum and lung for the prevention and clearance of infection (45). IgA alone has been shown to be sufficient to prevent influenza transmission (46), a proxy for decreasing infection severity. The dampening of the IgA response by alum, which could decrease the effectiveness of the vaccine at generating a protective humoral immune response, may explain its substandard performance as an adjuvant in influenza vaccination in this and previous vaccine trials $(11,41)$. Additionally, because MF59 
did not enhance the IgA levels as compared with unadjuvanted vaccination (Figure $2 \mathrm{C}$ and Supplemental Figure 2, E and F), this may also be an area of concern for future influenza vaccination regimens using MF59 to boost immunity, if this critical protective isotype is not induced at sufficient levels.

Beyond neutralization and HAI, many antibody functions have been implicated in protection from influenza infection and clearance after infection. Complement activation has been implicated in protection from influenza pathogenesis in mouse models $(16,47)$ and was transiently boosted with MF59 in our study (Figure $3 \mathrm{~F}$ ). Additionally, neutrophils also play a role in the phagocytic clearance of influenza-infected lung cells (48). Similarly, macrophage activity in the lung has been linked to protection from lethal influenza infection in mice (22), and human serum is known to have ADCP activity against circulating influenza strains that can clear virus and virally infected cells (49). MF59-induced increases in ADCP did not occur in a titer-dependent manner, although significantly increased ADCP activity persisted to day 208 (Figure 4A). Importantly, IgG-mediated neutrophil-mediated phagocytosis occurs in a largely FCGR2A-dependent manner (35), whereas monocytes are able to phagocytose in both an FCGR2A- and FCGR3A-dependent manner (35). Thus, compromised monocyte function, but not neutrophil phagocytic function, is likely related to the inability of MF59-induced antibodies to bind to FCGR3A (Figure 5B). These data point to enhanced clearance of ADNP antibodies, which may solely function through FCGR2A, and to the potential slower clearance of antibodies potentially harboring distinct Fc-glycan profiles able to drive ADCP through noncanonical Fc-receptors, such as lectin-like receptors, known to drive monocyte-mediated activity (50). Further, the correlations between functional and neutralizing antibodies become unlinked as the response wanes (Supplemental Figure 3). Defining the unique antibody subpopulations and their functional durability may provide important insights for next-generation long-lived vaccine design. However, despite the significantly increased titers and function of influenza-specific antibodies, MF59 showed poorly enhanced protection in clinical trials $(12,13)$, raising the possibility that these functions alone may not be sufficient for generalized protection from influenza.

Antibody-driven NK cell function has been implicated in protection against multiple infections, including $\operatorname{HIV}(51,52)$, malaria $(53,54)$, and Ebola (55). Likewise, while the protective role of NK cells in influenza is not fully understood, emerging data point to a critical role for NK cells in the protection against severe disease and the clearance of influenza infection $(15,29,56)$. The importance of NK cells has emerged in relationship to the disconnect wherein some broadly protective antibodies show limited HAI activity (19). Specifically, antibodies that bind to the stalk region of influenza HA and are protective in animal models require FCGR interactions to provide their protective effect, regardless of in vitro neutralization capability (18). Further, only stalkspecific and broadly reactive head-specific monoclonal antibodies, not head-targeting strain-specific mAbs, were shown to mediate ADCC (18). Recent studies in this field suggest that stalkspecific, but not head-specific, antibodies can mediate ADCC by NK cells because of a requirement for contact between the antibody Fc and the receptor-binding site of HA with sialic acids on the NK cell $(57,58)$. Strain-specific head-targeting antibodies typically block the receptor-binding-site interactions with sialic acid needed to efficiently induce ADCC (57). Owing to this involvement of NK cells in HA stalk-specific antibody-mediated protection, it has been speculated that NK cell activation may represent a vital Fc-effector function in broadly reactive humoral immunity against influenza. However, MF59 was unable to efficiently promote the development of NK cell-activating, FCGR3A-binding antibodies (Figure 4, C-E).

Monocytes and NK cells may mediate protection through multiple mechanisms. After the recognition of antibody-opsonized cells or virus, monocytes and/or NK cells may contribute to direct lysis and destruction, clearance of influenza-infected cells, or to the indirect arming of antiviral immunity via the release of IFN- $\gamma$ required to potentiate $\mathrm{T}$ cell immunity and efficient antigen uptake and activation of pulmonary dendritic cells (59). Recent data and opinion in the field has suggested a particular role for ADCC- or FCGR3A-binding antibodies (33); studies in mice have found protection by ADCC-inducing antibodies and correlations in human studies between ADCC titers $(18,60)$ and reductions in disease burden (61). FCGR3A is the primary Fc-receptor expressed on NK cells, responsible for NK cell activation $(62,63)$, but it is also expressed on mature macrophages (35). Affinity for binding to this receptor is influenced by the selection of antibody subclass and Fc glycosylation (35). Surprisingly, although MF59 induced higher levels of IgG1 and IgG3, known to bind to FCGR3A, MF59 did not induce antibodies able to enhance binding to FCGR3A (Figure 5B) or drive enhanced NK cell activation (Figure 4, C-E). The consistent deficit in FCGR3A and incomplete NK cell or monocyte responsiveness suggests that the induced IgG3 antibodies, which typically exhibit enhanced affinity for FCGR3A, may be functionally distinct from typical IgG3 responses, thought classically to drive enhanced ADCC activity. However, like IgG1 antibodies, IgG3 also possess an Fc-glycan, which may also change after vaccination and infection, potentially resulting in differential affinity for Fc-receptors. Thus, future studies focused selectively on IgG3 glycosylation differences across adjuvant platforms may point to opportunities to shape overall and subclass-specific antibody functionality.

Preexisting antibodies from previous influenza exposure or vaccination have been an issue in the development of influenza vaccination, both seasonal and pandemic, and cross-reactive antibodies to conserved epitopes such as the HA stem have been considered a possible benefit $(37,64)$. Preexisting antibodies have been shown to influence the magnitude, epitope targeting, and neutralizing capacity of vaccine-induced immunity, yet it has been uncertain whether preexisting immunity could also influence antibody effector function. Here, we observed limited evidence of an influence of preexisting $\mathrm{H} 1$ responses on $\mathrm{H} 5$-induced antibody functionality (Figure 6), which was induced equivalently with MF59 across groups. The influence of stem-specific preexisting responses, which are likely to interact more readily with $\mathrm{H} 5$, was not investigated here, but these responses could potentially be leveraged to drive enhanced immunity to this diverging strain. Thus, future studies focused on the interrogation of epitope-specific functional responses may uncover additional roles for preexisting antibodies on shaping vaccine-induced immunity. 
The robust induction of antibody titers and neutralization using MF59 has resulted in only a modest increase in protection against seasonal and pandemic influenza in vulnerable populations (12, 13), although the effectiveness of the adjuvant against human avian infections has yet to be studied. This disconnect may be related to the ability of MF59 to significantly augment neutralization and antibody function that appears to harbor a functional "hole" in NK cell and monocyte activation (Figure 3). Given our emerging appreciation for opportunities to combine adjuvants because of their complementary immune-stimulating capabilities $(44,65,66)$, defining the landscape of qualitative modifications induced by individual and combined adjuvants may offer an opportunity to fully control the quantity and quality of the humoral immune response. Thus, with our growing appreciation for the importance of particular Fc-effector functions across infections (15, 51-55), next-generation rational vaccine design efforts may benefit from a comprehensive understanding of the functional impact and opportunities to conditionally elicit vaccine-induced antibody effector function to maximize the potency of the antigen-binding and constant domaindriven function of vaccine-induced antibodies.

\section{Methods}

Vaccine schedule and sample collection. Samples were collected at the National Institute of Allergy and Infectious Diseases-funded (NIAID-funded) Vaccine and Treatment Evaluation Units (ClinicalTrials.gov NCT00280033). The results of this trial have been published previously (11). H5N1-naive adult study participants (ages 18-64) provided informed consent and were then randomly assigned to receive $15 \mu \mathrm{g}$ recombinant $\mathrm{H} 5$ A/Vietnam/1203/2004 antigen without adjuvant, with alum, or with MF59 in 2 doses administered 28 days apart. Samples from 90 individuals ( 30 without adjuvant, 29 with alum, 31 with MF59) at 4 time points $(0,28,56$, and 208 days after the initial dose) were assayed.

Antibody-dependent cellular phagocytosis. THP-1 phagocytosis of HA-coated beads was performed as previously described (67). Briefly, H5 A/Vietnam/1203/2004 (Immune Technology Corp.) or H1 A/New Caledonia/20/1999 (Immune Technology Corp.) was biotinylated with EZ-link NHS-LC-LC Biotin per the manufacturer's instructions (Thermo Fisher Scientific). Biotinylated HA was adsorbed onto $1 \mu \mathrm{m}$ fluorescent neutravidin beads at a ratio of $10 \mu \mathrm{g}$ of protein to $10 \mu \mathrm{l}$ of beads (Invitrogen). Then, $10 \mu \mathrm{l}$ of antigen-coated beads was incubated with an equal volume of serum samples diluted in PBS for 2 hours at $37^{\circ} \mathrm{C}$ in 96 -well plates. Unbound antibody was washed away, and THP- 1 cells (ATCC) were added and incubated at $37^{\circ} \mathrm{C}$ for 16 hours and then fixed. Phagocytosis was measured by flow cytometry on a BD LSRII cytometer with a high-throughput sampler. Phagocytic scores were calculated as the percentage of bead-positive cells times the geometric mean fluorescence intensity (GMFI)/1,000. Area under the curve was calculated using GraphPad Prism based on 3 dilutions: 1:50, 1:500, and 1:5,000. Each sample was assayed in 2 independent replicates performed on different days. Quality checks were performed to ensure that known positive samples were at least 2 times higher than negative controls and that replicate values correlated. After the quality checks, values from replicates were averaged.

Antibody-dependent neutrophil phagocytosis. HA-coated $1 \mu \mathrm{m}$ fluorescent neutravidin beads were prepared and incubated with an equal volume of diluted serum samples, as in the ADCP assay. For
ADNP, optimal dilution (1:50) was predetermined by titration. Primary human leukocytes were isolated from healthy donors using ammoniumchloride potassium lysis, added to the opsonized beads, and incubated at $37^{\circ} \mathrm{C}$ for 1 hour. The cells were then stained with fluorescent antihuman CD66b (Biolegend, catalog no. 305112) and fixed prior to analysis on a BD LSRII cytometer with a high-throughput sampler. Phagocytic scores were calculated as the percentage of bead CD $66^{+}$cells times GMFI/1,000. Two healthy leukocyte donors were used to assay each sample in parallel. Quality checks were performed to ensure that known positive samples were at least 2 times higher than negative controls and that replicate values correlated. After the quality checks, values from replicates were averaged.

Antibody-dependent complement deposition. Serum samples were heat inactivated at $56^{\circ} \mathrm{C}$ for 30 minutes and centrifuged to remove aggregates. HA-coated $1 \mu \mathrm{m}$ fluorescent neutravidin beads were prepared as in the ADCP assay and incubated with diluted, heat-inactivated serum samples for 20 minutes at $37^{\circ} \mathrm{C}$. Lyophilized guinea pig complement (Cedarlane) was resuspended in ice-cold water, then diluted 1:60 in veronal buffer with $0.1 \%$ gelatin added. Next, $150 \mu \mathrm{l}$ diluted complement was added to the opsonized beads and incubated for 20 minutes at $37^{\circ} \mathrm{C}$. Beads were then washed with $15 \mathrm{mM}$ EDTA and stained with anti-guinea pig C3 (MP Biomedicals, catalog no. 855385). Samples were washed and analyzed on a BD LSRII cytometer with a high-throughput sampler. Complement scores were the percentage of $\mathrm{C}^{+}$beads times GMFI/10,000. Area under the curve was calculated using GraphPad Prism based on 3 dilutions: 1:15, 1:30, and 1:60. Each sample was assayed in 2 independent replicates performed on different days. Quality checks were performed to ensure that known positive samples were at least 2 times higher than negative controls, and that replicate values correlated. After the quality checks, values from replicates were averaged.

Antibody-dependent NK cell activation. Antibody-dependent NK cell activation and degranulation were measured as previously described $(68,69)$. ELISA plates (Thermo Fisher Scientific NUNC MaxiSorp) were coated with H5 A/Vietnam/1203/2004 and then blocked. Serum samples were then diluted 1:60 (dilution predetermined by titration) and $100 \mu$ ladded to each well and incubated for 2 hours at $37^{\circ} \mathrm{C}$. NK cells were isolated from buffy coats from healthy donors using the RosetteSep NK cell enrichment kit (STEMCELL Technologies) and rested in $1 \mathrm{ng} / \mathrm{ml} \mathrm{IL-15}$ at $37^{\circ} \mathrm{C}$ until needed. NK cells, with anti-CD107a PE-Cy5 (BD, catalog no. 555802), brefeldin A (Sigma-Aldrich), and GolgiStop (BD), were added and incubated for 5 hours at $37^{\circ} \mathrm{C}$. Cells were stained for surface markers using antiCD56 PE-Cy7 (BD, catalog no. 557747), anti-CD16 APC-Cy7 (BD, catalog no. 557758), and anti-CD3 AF700 (BD, catalog no. 557943), then fixed and permeabilized using FIX \& PERM Cell Permeabilization Kit (Thermo Fisher Scientific). Cells were stained for intracellular markers using anti-MIP1 $\beta$ PE (BD, catalog no. 550078) and anti-IFN- $\gamma$ APC (BD, catalog no. 554702). Fixed cells were analyzed by flow cytometry on a BD LSRII equipped with a high-throughput sampler. NK cells were defined as $\mathrm{CD}^{-}$and $\mathrm{CD} 16 / 56^{+}$. Two healthy NK cell donors were used to assay each sample in parallel. Quality checks were performed to ensure that known positive samples were at least 2 times higher than negative controls, and that replicate values correlated. After the quality checks, values from replicates were averaged.

Ig subclassing/isotyping and FCR binding. Antigen-specific antibody subclass/isotypes and FcR binding were determined using a 
high-throughput Luminex-based assay, which has been shown to correlate highly with more laborious SPR techniques (70). Antigens included were the following: H1 HA A/New Caledonia/20/99, H3 HA A/Brisbane/10/2007, H5 A/Vietnam/1203/2004, HA B/Florida/ 4/2006, EBV recombinant gH(D1-111)/gL/gp42 (Ectodomain) (Strain B95-8), all provided from Immune Technology Corp., and EBOV GPdTM from Mayflower Bioscience, St. Louis, MO. Antigens were coupled to carboxylate-modified microspheres (Luminex Corp.) by covalent NHS-ester linkages via EDC (Thermo Fisher Scientific) and Sulfo-NHS (Thermo Fisher Scientific) per the manufacturer's instructions. These antigen-coated microspheres were added to nonbinding 384-well plates (Grenier Bio-One) at 1,000 beads per well (45 $\mu \mathrm{l})$. Serum samples were diluted 1:100 for IgG1, total IgG and all FcRs, and 1:10 for other antibody subclasses. Next, $5 \mu \mathrm{l}$ of diluted serum samples were added and incubated with microspheres on a shaker for 2 hours at room temperature or overnight at $4^{\circ} \mathrm{C}$. Sample dilutions were determined by assaying a dilution curve and selecting a dilution within the linear range of the assay. Microspheres were washed, and PE-conjugated anti-IgG1 (catalog no. 9052-09), anti-IgG2 (catalog no. 9060-09), anti-IgG3 (catalog no. 9210-09), anti-IgG4 (catalog no. 9200-09), anti-IgG (catalog no. 9040-09), anti-IgM (catalog no. 9020-09), anti-IgA1 (catalog no. 9130-09), or anti-IgA2 (catalog no. 9140-09) detection antibodies (all Southern Biotech) or biotinylated FcRs (Duke University Protein Production Core) conjugated to streptavidin-PE (Prozyme) were added for 1 hour at room temperature. The microspheres were washed and read on a Bio-Plex 3D system (Luminex Corp.) or iQue Plus screener (Intellicyt). All samples were assayed in duplicate and values were averaged. Data were screened to ensure adequate bead counts $(>20)$ per antigen per sample, and values for sample/antigen pairs with inadequate counts were excluded from further analyses.

Statistics. For PCA, data were normalized using Box-Cox transformation and $Z$-scored. Missing values were imputed using k-nearest neighbor. PCA was performed to resolve differences between vacci- nation groups using all measured features as inputs using MATLAB (MathWorks). To compare the difference between 2 groups (none vs. MF59 or alum vs. MF59) of nonnormally distributed PC1 values, Mann-Whitney $U$ tests were calculated with GraphPad Prism.

For univariate analyses comparing multiple groups, ANOVA followed by Tukey's multiple comparison test was used; a $P$ value of 0.05 was considered significant. For correlations, Spearman's rank correlation coefficient was used; a $P$ value of 0.05 was considered significant.

Study approval. Written informed consent was obtained before inclusion of subjects in the MF59 vaccine trial (11). The current work was approved by Massachusetts General Hospital Institutional Review Board (approval 2015P000646).

\section{Author contributions}

CMB, TS, and GA designed the research study. CMB conducted the experiments and acquired data. CMB and WHY analyzed data. HKT and KE provided study samples. CMB, TS, and GA wrote the manuscript. All authors contributed to the final version of the manuscript.

\section{Acknowledgments}

We thank C. Boggiano at the NIAID for assistance in procuring samples, and A. Butler, E. Irvine, M. Jennewein, and S. Fischinger for discussion and feedback. This work was supported by the following: the Ragon Institute, the Samana Cay Massachusetts General Hospital scholar program, and NIH grants AI080289 (5R37AI080289-09), AI102660-01 (5R01AI102660-04), and AI129797-01 (1R01A1129797-01), as well as NIH T32 AI007245 (CMB). We would also like to acknowledge Harvard CFAR for ongoing support through P30 AI060354-02.

Address correspondence to: Galit Alter, 400 Technology Square, Room 784, Cambridge, Massachusetts 02139, USA. Phone: 857.268.7003; Email: galter@mgh.harvard.edu.
1. Iuliano AD, et al. Estimates of global seasonal influenza-associated respiratory mortality: a modelling study. Lancet. 2018;391(10127):1285-1300.

2. CDC. Disease Burden of Influenza. CDC website http://www.cdc.gov/flu/about/burden/index. html. Accessed November 13, 2019.

3. WHO. H5N1 avian influenza: Timeline of major events 2012. http://www.who.int/influenza/ human_animal_interface/H5N1_avian_influenza_update200412.pdf. WHO website. Updated April 20, 2012. Accessed November 13, 2019.

4. WHO Global Influenza Programme. Avian Influenza: Assessing The Pandemic Threat. Geneva, Switzerland:WHO; 2005.

5. Banzhoff A, et al. MF59-adjuvanted H5N1 vaccine induces immunologic memory and heterotypic antibody responses in non-elderly and elderly adults. PLoS One. 2009;4(2):e4384.

6. Coffman RL, Sher A, Seder RA. Vaccine adjuvants: putting innate immunity to work. Immunity. 2010;33(4):492-503.

7. Tregoning JS, Russell RF, Kinnear E. Adjuvanted influenza vaccines. Hum Vaccin Immunother. 2018;14(3):550-564.

8. CDC. FLUAD Flu Vaccine with Adjuvant 2017. 13. https://www.cdc.gov/flu/prevent/adjuvant.htm. 9. Seubert A, Monaci E, Pizza M, O’Hagan DT, Wack A. The adjuvants aluminum hydroxide and MF59 induce monocyte and granulocyte chemoattractants and enhance monocyte differentiation toward dendritic cells. J Immunol. 2008;180(8):5402-5412.

10. Khurana S, et al. MF59 adjuvant enhances diversity and affinity of antibody-mediated immune response to pandemic influenza vaccines. $\mathrm{Sci}$ Transl Med. 2011;3(85):85ra48.

11. Bernstein DI, et al. Effects of adjuvants on the safety and immunogenicity of an avian influenza H5N1 vaccine in adults. J Infect Dis. 2008;197(5):667-675.

12. Vesikari T, et al. Efficacy, immunogenicity, and safety evaluation of an MF59-adjuvanted quadrivalent influenza virus vaccine compared with non-adjuvanted influenza vaccine in children: a multicentre, randomised controlled, observer-blinded, phase 3 trial. Lancet Respir Med.2018;6(5):345-356.

13. Domnich A, Arata L, Amicizia D, Puig-Barberà J, Gasparini R, Panatto D. Effectiveness of MF59adjuvanted seasonal influenza vaccine in the elderly: A systematic review and meta-analysis.
Vaccine. 2017;35(4):513-520

14. Wong SS, et al. The immune correlates of protection for an avian influenza $\mathrm{H} 5 \mathrm{~N} 1$ vaccine in the ferret model using oil-in-water adjuvants. Sci Rep. 2017;7:44727.

15. Jegaskanda S, Vanderven HA, Wheatley AK, Kent SJ. Fc or not Fc; that is the question: antibody Fc-receptor interactions are key to universal influenza vaccine design. Hum Vaccin Immunother. 2017;13(6):1-9.

16. O'Brien KB, Morrison TE, Dundore DY, Heise MT, Schultz-Cherry S. A protective role for complement C3 protein during pandemic 2009 H1N1 and $\mathrm{H} 5 \mathrm{~N} 1$ influenza A virus infection. PLoS One. 2011;6(3):e17377.

17. Rattan A, et al. Synergy between the classical and alternative pathways of complement is essential for conferring effective protection against the pandemic influenza A(H1N1) 2009 virus infection. PLoS Pathog. 2017;13(3):e1006248.

18. DiLillo DJ, Tan GS, Palese P, Ravetch JV. Broadly neutralizing hemagglutinin stalkspecific antibodies require $\mathrm{F} c \gamma \mathrm{R}$ interactions for protection against influenza virus in vivo. $\mathrm{Nat}$ Med. 2014;20(2):143-151. 
19. DiLillo DJ, Palese P, Wilson PC, Ravetch JV. Broadly neutralizing anti-influenza antibodies require $\mathrm{Fc}$ receptor engagement for in vivo protection. J Clin Invest. 2016;126(2):605-610.

20. Mullarkey CE, et al. Broadly neutralizing hemagglutinin stalk-specific antibodies induce potent phagocytosis of immune complexes by neutrophils in an Fc-dependent manner. MBio. 2016;7(5):e01624-16.

21. Huber VC, Lynch JM, Bucher DJ, Le J, Metzger DW. Fc receptor-mediated phagocytosis makes a significant contribution to clearance of influenza virus infections. JImmunol. 2001;166(12):7381-7388.

22. He W, et al. Alveolar macrophages are critical for broadly-reactive antibody-mediated protection against influenza A virus in mice. Nat Commun. 2017;8(1):846

23. Terajima M, Co MD, Cruz J, Ennis FA. High antibody-dependent cellular cytotoxicity antibody titers to H5N1 and H7N9 avian influenza a viruses in healthy US adults and older children. J Infect Dis. 2015;212(7):1052-1060.

24. Lu LL, Suscovich TJ, Fortune SM, Alter G. Beyond binding: antibody effector functions in infectious diseases. Nat Rev Immunol. 2018;18(1):46-61.

25. Gunn BM, Alter G. Modulating antibody functionality in infectious disease and vaccination. Trends Mol Med. 2016;22(11):969-982.

26. Ackerman ME, et al. Polyfunctional HIVspecific antibody responses are associated with spontaneous HIV control. PLoS Pathog. 2016;12(1):e1005315.

27. Jegaskanda S. The potential role of Fc-receptor functions in the development of a universal influenza vaccine. Vaccines (Basel). 2018;6(2):E27.

28. Kopf M, Abel B, Gallimore A, Carroll M, Bachmann MF. Complement component C3 promotes T-cell priming and lung migration to control acute influenza virus infection. Nat Med. 2002;8(4):373-378.

29. Vanderven HA, et al. Antibody-dependent cellular cytotoxicity responses to seasonal influenza vaccination in older adults. J Infect Dis. 2017;217(1):12-23.

30. Chung AW, et al. Dissecting polyclonal vaccineinduced humoral immunity against HIV using systems serology. Cell. 2015;163(4):988-998.

31. Kao D, Lux A, Schaffert A, Lang R, Altmann F, Nimmerjahn F. IgG subclass and vaccination stimulus determine changes in antigen specific antibody glycosylation in mice. Eur J Immunol. 2017;47(12):2070-2079.

32. O'Hagan DT, Rappuoli R, De Gregorio E, Tsai T, Del Giudice G. MF59 adjuvant: the best insurance against influenza strain diversity. Expert Rev Vaccines. 2011;10(4):447-462.

33. Jegaskanda S, Reading PC, Kent SJ. Influenza-specific antibody-dependent cellular cytotoxicity: toward a universal influenza vaccine. J Immunol. 2014;193(2):469-475.

34. Vanderven HA, Jegaskanda S, Wheatley AK, Kent SJ. Antibody-dependent cellular cytotoxicity and influenza virus. Curr Opin Virol. 2017;22:89-96.

35. Bournazos S, Woof JM, Hart SP, Dransfield I. Functional and clinical consequences of Fc receptor polymorphic and copy number variants. Clin Exp Immunol. 2009;157(2):244-254.
36. Bruhns P, et al. Specificity and affinity of human $\mathrm{Fc} \gamma$ receptors and their polymorphic variants for human IgG subclasses. Blood. 2009;113(16):3716-3725.

37. Zhang A, Stacey HD, Mullarkey CE, Miller MS. Original antigenic sin: how first exposure shapes lifelong anti-influenza virus immune responses. J Immunol. 2019;202(2):335-340.

38. Lambert LC, Fauci AS. Influenza vaccines for the future. N Engl JMed. 2010;363(21):2036-2044

39. Ren H, Zhou P. Epitope-focused vaccine design against influenza A and B viruses. Curr Opin Immunol. 2016;42:83-90.

40. Houser K, Subbarao K. Influenza vaccines: challenges and solutions. Cell Host Microbe. 2015;17(3):295-300.

41. Ahmed SS, Plotkin SA, Black S, Coffman RL. Assessing the safety of adjuvanted vaccines. Sci Transl Med. 2011;3(93):93rv2.

42. Clegg CH, Rininger JA, Baldwin SL. Clinical vaccine development for H5N1 influenza. Expert Rev Vaccines. 2013;12(7):767-777.

43. Galli G, et al. Fast rise of broadly cross-reactive antibodies after boosting long-lived human memory B cells primed by an MF59 adjuvanted prepandemic vaccine. Proc Natl Acad Sci US A. 2009;106(19):7962-7967.

44. Francica JR, et al. Innate transcriptional effects by adjuvants on the magnitude, quality, and durability of HIV envelope responses in NHPs. Blood Adv. 2017;1(25):2329-2342.

45. Renegar KB, Small PA, Boykins LG, Wright PF. Role of IgA versus IgG in the control of influenza viral infection in the murine respiratory tract. J Immunol. 2004;173(3):1978-1986.

46. Seibert CW, et al. Recombinant IgA is sufficient to prevent influenza virus transmission in guinea pigs. J Virol. 2013;87(14):7793-7804.

47. Hicks JT, Ennis FA, Kim E, Verbonitz M. The importance of an intact complement pathway in recovery from a primary viral infection: influenza in decomplemented and in C5-deficient mice. Jimmunol. 1978;121(4):1437-1445.

48. Hashimoto Y, Moki T, Takizawa T, Shiratsuchi A, Nakanishi Y. Evidence for phagocytosis of influenza virus-infected, apoptotic cells by neutrophils and macrophages in mice. JImmunol. 2007;178(4):2448-2457.

49. Ana-Sosa-Batiz F, et al. Influenza-specific antibody-dependent phagocytosis. PLoS One. 2016;11(4):e0154461.

50. Kerrigan AM, Brown GD. C-type lectins and phagocytosis. Immunobiology. 2009;214(7):562-575.

51. Barouch DH, et al. Protective efficacy of a global HIV-1 mosaic vaccine against heterologous SHIV challenges in rhesus monkeys. Cell. 2013;155(3):531-539.

52. Chung AW, et al. Polyfunctional Fc-effector profiles mediated by IgG subclass selection distinguish RV144 and VAX003 vaccines. Sci Transl Med. 2014;6(228):228ra38.

53. Tiendrebeogo RW, et al. Antibody-dependent cellular inhibition is associated with reduced risk against febrile malaria in a longitudinal cohort study involving Ghanaian children. Open Forum Infect Dis. 2015;2(2):ofv044.

54. Jafarshad A, Dziegiel MH, Lundquist R, Nielsen
LK, Singh S, Druilhe PL. A novel antibodydependent cellular cytotoxicity mechanism involved in defense against malaria requires costimulation of monocytes Fc $\gamma$ RII and Fc $\gamma$ RIII. JImmunol. 2007;178(5):3099-3106.

55. Gunn BM, et al. A role for Fc function in therapeutic monoclonal antibody-mediated protection against Ebola virus. Cell Host Microbe. 2018;24(2):221-233.e5.

56. Jegaskanda S, et al. Cross-reactive influenzaspecific antibody-dependent cellular cytotoxicity antibodies in the absence of neutralizing antibodies. JImmunol. 2013;190(4):1837-1848.

57. Cox F, et al. HA antibody-mediated Fc $\gamma$ RIIIa activity is both dependent on FcR engagement and interactions between HA and sialic acids. Front Immunol. 2016;7:399.

58. Leon PE, et al. Optimal activation of Fc-mediated effector functions by influenza virus hemagglutinin antibodies requires two points of contact. Proc Natl Acad Sci U S A. 2016;113(40):E5944-E5951.

59. Ge MQ, et al. NK cells regulate $\mathrm{CD} 8^{+} \mathrm{T}$ cell priming and dendritic cell migration during influenza A infection by IFN- $\gamma$ and perforin-dependent mechanisms. JImmunol. 2012;189(5):2099-2109.

60. Impagliazzo A, et al. A stable trimeric influenza hemagglutinin stem as a broadly protective immunogen. Science. 2015;349(6254):1301-1306.

61. Jegaskanda S, et al. Generation and protective ability of influenza virus-specific antibody-dependent cellular cytotoxicity in humans elicited by vaccination, natural infection, and experimental challenge. J Infect Dis. 2016;214(6):945-952.

62. Perussia B, et al. Human natural killer cells analyzed by B73.1, a monoclonal antibody blocking Fc receptor functions. II. Studies of B73.1 antibody-antigen interaction on the lymphocyte membrane. J Immunol. 1983;130(5):2142-2148.

63. Ritz J, Schmidt RE, Michon J, Hercend T, Schlossman SF. Characterization of functional surface structures on human natural killer cells. $A d v$ Immunol. 1988;42:181-211.

64. Kim JH, Skountzou I, Compans R, Jacob J. Original antigenic sin responses to influenza viruses. JImmunol. 2009;183(5):3294-3301.

65. Kasturi SP, et al. Programming the magnitude and persistence of antibody responses with innate immunity. Nature. 2011;470(7335):543-547.

66. Wack A, et al. Combination adjuvants for the induction of potent, long-lasting antibody and $\mathrm{T}$-cell responses to influenza vaccine in mice. Vaccine. 2008;26(4):552-561.

67. Ackerman ME, et al. A robust, high-throughput assay to determine the phagocytic activity of clinical antibody samples. JImmunol Methods. 2011;366(1-2):8-19.

68. Lu LL, et al. A functional role for antibodies in tuberculosis. Cell. 2016;167(2):433-443.e14.

69. Jegaskanda S, Weinfurter JT, Friedrich TC, Kent SJ. Antibody-dependent cellular cytotoxicity is associated with control of pandemic H1N1 influenza virus infection of macaques. J Virol. 2013;87(10):5512-5522.

70. Brown EP, et al. High-throughput, multiplexed IgG subclassing of antigen-specific antibodies from clinical samples. J Immunol Methods. 2012;386(1-2):117-123. 